\title{
GESTIÓN INTEGRADA PARA LA CUENCA TRANSFRONTERIZA DEL CHINCHIPE EN EL NORTE PERUANO
}

\section{INTEGRATED MANAGEMENT FOR THE CHINCHIPE TRANSBOUNDARY BASIN IN NORTHERN PERU}

\author{
(iD) Josué Otoniel Dilas-Jiménez ${ }^{1}$ \\ 'Universidad Nacional Autónoma de Tayacaja Daniel Hernández Morillo, Perú. \\ Correspondencia: \\ Mag. Josué Otoniel Dilas Jiménez \\ jdilas@unat.edu.pe
}

\section{RESUMEN}

La gestión integrada de la cuenca debe ser analizada desde un punto de vista complejo y desde las distintas ópticas que lo abordan. La implementación de acciones y planes de gestión integrada de cuencas no es sencillo, dada la alta variabilidad del territorio en el caso peruano, sobre todo cuando se trata de cuencas transfronterizas. La cuenca del Chinchipe, cuenca transfronteriza Perú-Ecuador, es parte de la vertiente amazónica, se ubica en una zona estratégica y productiva del norte peruano principalmente en territorio de la región Cajamarca, predomina la actividad agrícola con cultivo de café en la cuenca media y cultivos de arroz, cacao, otros cultivos en la cuenca baja. Entre sus principales debilidades para la gestión integrada se destaca la no conformación del Consejo de Recurso Hídricos de la Cuenca como actor institucional básico del sistema según la Ley peruana, el limitado empoderamiento de la Autoridad Local del Agua Chinchipe-Chamaya, la escasa integración de los actores de la cuenca, entre otros. Sin embargo, se ha analizado que esta cuenca tiene un alto potencial para contribuir al desarrollo del territorio, por ello, en este documento se desarrolla una propuesta de gestión integrada de la cuenca del Chinchipe.

Palabras clave: San Ignacio, Chinchipe, cuenca, gestión integrada, recursos hídricos.

\section{ABSTRACT}

The integrated management of the watershed must be analyzed from a complex point of view and from the different perspectives that address it. The implementation of actions and plans for integrated watershed management is not easy given the high variability of the territory in the Peruvian case, especially when it comes to transboundary basin. The Chinchipe basin, Peru-Ecuador transboundary basin, is part of the Amazonian slope, it is located in a strategic and productive area of northern Peru, mainly in the territory of the Cajamarca region, and agricultural activity with coffee cultivation predominates in the middle basin and rice crops, cocoa, other crops in the lower basin. Among its main weaknesses for integrated management, the nonconformation of the Watershed Water Resources Council as the basic institutional actor of the system according to Peruvian law, the limited empowerment of the 
Chinchipe-Chamaya Local Water Authority, the scarce integration of the basin actors, among others. However, it has been analyzed that this basin has a high potential to contribute to the development of the territory, therefore, this document develops a proposal for integrated management of the Chinchipe basin.

Key words: San Ignacio, Chinchipe, basin, integrated management, water resources

\section{INTRODUCCIÓN}

Como espacio geográfico o unidad territorial, una cuenca hidrográfica se entiende como un territorio delimitado geográficamente por la divisoria de aguas (lluvias, deshielos, otros) que se juntan en riachuelos y quebradas que forman luego un cauce principal o rio que llega hacia un único lugar o punto de descarga que puede ser un rio o lago u océano (Felipe-Morales, 2007; Aguirre, 2011). Según la CEPAL el manejo de cuencas se puede definir como "la gestión que el hombre realiza a nivel de esta área para aprovechar y proteger los recursos naturales que le ofrece, con el fin de obtener una producción óptima y sostenida", así, entre las alternativas de manejo se puede considerar a los proyectos de aprovechamiento hídrico (hidroeléctricas), de manejo de bosques, de desarrollo agrario, de riego, entre otros (CEPAL, 1982). Por su parte Faustino \& Jiménez (2000), precisan que el manejo de cuencas puede relacionarse con el manejo apropiado que el hombre da a los recursos de la cuenca para satisfacer sus necesidades, propiciando la sostenibilidad, la calidad de vida y el desarrollo.

Por otro lado, desde el enfoque de sistemas, una cuenca se comporta como un sistema ya que conforma componentes físicos o abióticos (clima, relieve, suelo) y componentes bióticos (vegetación, animales, microorganismos, seres humanos) que se interrelacionan entré sí, por lo que cualquier cambio que ocurriera en alguno de ellos, puede afectar a todo el sistema de la cuenca, de ahí nace la propuesta de "gestión integrada de cuencas" (Felipe-Morales, 2007). Entonces, para implementar una propuesta de gestión integrada de cuencas es necesario realizar una caracterización de la cuenca teniendo cuenta al menos lo siguiente (Faustino \& Jiménez, 2000): 1) Diagnóstico biofísico: vinculado a la evaluación el estado situacional de la cuenca en cuanto a sus recursos naturales; 2) Diagnóstico socioeconómico: aspectos sociales, económicos, culturales, entre otros. Para tal efecto, el análisis y las propuestas de manejo podrán diferenciarse teniendo en cuenta la caracterización que la cuenta pueda tener según la subdivisión por altitud (Felipe-Morales, 2007) como cuenca alta, cuenca media y cuenca baja.

Así, a nivel del mundo, desde décadas atrás se viene promoviendo la estrategia de desarrollo del territorio con enfoque de cuencas (Dourojeanni, 1994; Dourojeanni, Jouravlev \& Chávez, 2002). En el Perú según la Autoridad Nacional del Agua (ANA), se tienen identificadas 159 unidades hidrográficas las cuales se agrupan en tres grandes vertientes hidrográficas: la vertiente hidrográfica del Pacífico con 62 unidades; la vertiente hidrográfica del Amazonas con 84 unidades y la vertiente hidrográfica del Titicaca con 13 unidades (ANA, 2009). Sin embargo, es poco el avance que se ha tenido en cuanto a la implementación de estrategias de gestión integrada de cuencas, siendo así que a la actualidad en todo el Perú se tiene reconocidos sólo 12 Consejos de Recursos Hídricos de Cuenca y otros tres en proceso de reconocimiento (ANA, 2020b). Más aún, cuando se trata de hacer intervenciones en cuencas transfronterizas, identificadas en el Perú en un número de 34 (ANA, 2015), entre ellas, la cuenca del Chinchipe ubicada en territorio del Perú y del Ecuador, remarca un alto interés su adecuada gestión, ya que su territorio en el lado peruano representa importante desarrollo económico productivo de la región Cajamarca, también alberga importante flora, fauna y tiene ecosistemas de bosque montano de neblina altamente eficientes en captura de carbono (Elliot, 2009; Llerena et al., 2010; Dilas-Jiménez \& Huamán Jiménez, 2020). 
El objetivo planteado en el presente trabajo es realizar una caracterización general de la cuenca transfronteriza del Chinchipe y proponer un modelo de gestión integrada de esta cuenca, puntualmente en la parte del territorio peruano.

\section{MATERIALES Y MÉTODOS}

La caracterización se realizó mediante búsqueda y sistematización de información secundaria publicada sobre el territorio de la cuenca del Chinchipe.

Para la propuesta de gestión integrada de la cuenca del Chinchipe se utilizó la metodología del tablero de gestión integrada de cuencas propuesta por Dourojeanni $(2000,2020)$ que involucra un análisis de:

1) Las políticas, 2) Los actores, 3) Los instrumentos de gestión de intervenciones sobre el agua y sus cuencas de captación y 4) Los problemas y soluciones vinculadas al agua en cada cuenca; y como algo transversal la gobernanza de la cuenca.

\section{DESARROLLO}

\section{Caracterización biofísica de la cuenca del Chinchipe. \\ Ubicación e hidrografía de la cuenca}

De las 159 cuencas o unidades hidrográficas identificadas por la Autoridad Nacional del Agua (ANA) en el Perú (ANA, 2015), la cuenca del rio Chinchipe corresponde a la unidad hidrográfica 114 con el código 49892 (ANA, 2009), desemboca en la gran cuenca del rio Marañón (cuenca del alto Marañón), esto en la vertiente hidrográfica del Amazonas. Cabe precisar que la gran cuenca del rio Marañón se subdivide en dos cuencas: cuenca del Alto Marañón (desde su naciente en el nevado Raura-Huánuco hasta el pongo de Manseriche en el límite de amazonas-Loreto) y cuenca del Bajo Marañón (desde el pongo de Manseriche hasta el punto de confluencia con el rio Ucayali para desembocar en el gran rio Amazonas) (Carranza, 2011).
Entonces, la cuenca del Chinchipe, es una cuenca transfronteriza ya que abarca territorio de Ecuador (sur) y Perú (norte), siendo así una de las 34 cuencas transfronterizas identificadas en el Perú (ANA, 2015). Su naciente es en la cordillera de Sabanilla en la provincia de Zamora (cantón Chinchipe y cantón Palanda) al sur de ecuador, en cuyo cause principal convergen los ríos Palanda y Numbala, luego, cerca de la línea fronteriza con el Perú se unen los ríos Mayo y Canchis formando así el río Chinchipe, este continua en el territorio peruano donde se unen los ríos Chirinos y Tabaconas, así hasta su desembocadura en el río Marañón. En toda su extensión esta cuenca abarca 9686 km2, de los cuales 6538 km2 en el territorio peruano y $3148 \mathrm{~km} 2$ en el territorio ecuatoriano (Elliot, 2009; Vasquez, 2011). Según la ANA la extensión de esta cuenca recorre unos $142 \mathrm{~km}$ de cauce principal (ANA, 2011).

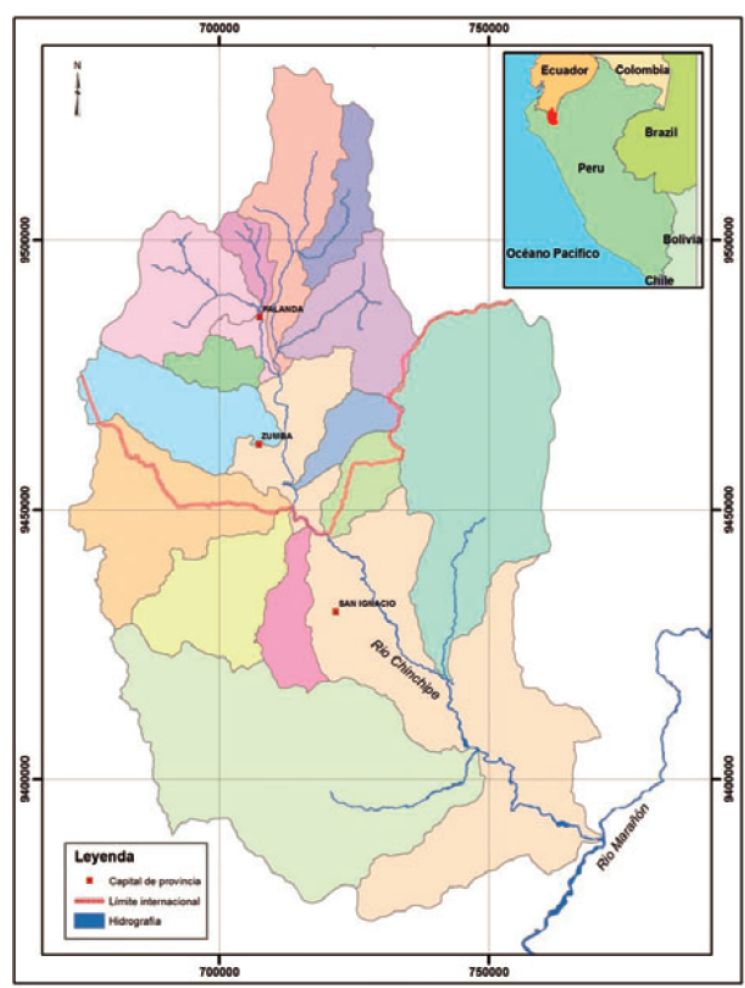

Figura 1. Ubicación y extensión de la cuenca del Chinchipe (Llerena et al., 2010) 


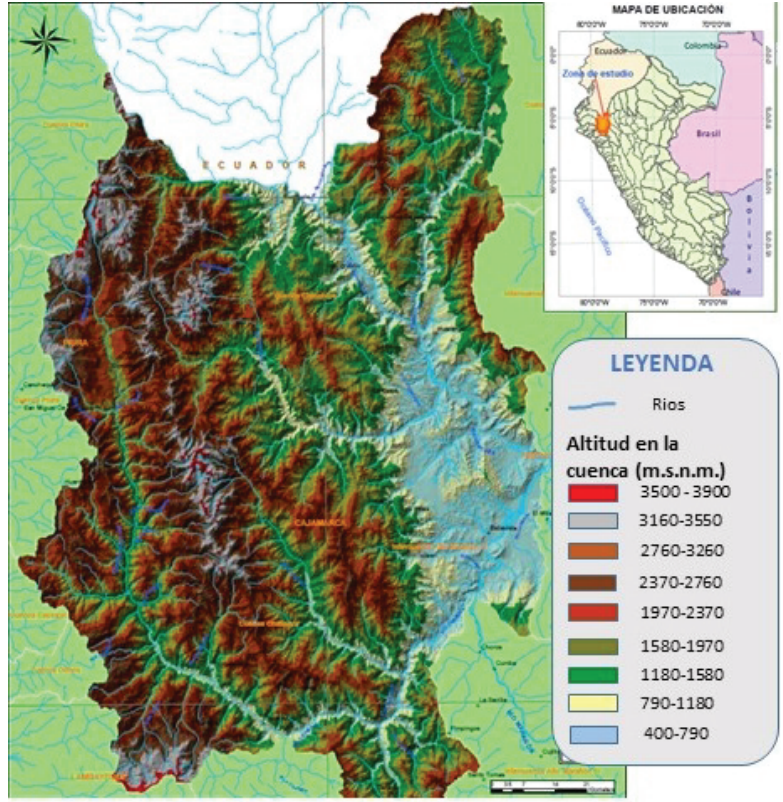

Figura 2. Mapa de la cuenca según altitudes (m.s.n.m) en la parte peruana. Fuente (ANA, 2011)

En el territorio peruano, esta cuenca abarca principalmente territorio de la región Cajamarca, puntualmente parte de la provincia de Jaén y la provincia de San Ignacio, ésta última representa la mayor parte del territorio de la cuenca (68\%); así también, abarca parte del departamento de Piura (Elliot, 2009).

\section{Características en la cuenca alta, cuenca media y cuenca baja}

Atendiendo a una caracterización del territorio de la cuenca según altitud (Felipe-Morales, 2007), la cuenca del Chinchipe se caracteriza por (Elliot, 2009; Llerena et al., 2010):

a) Cuenca alta del Chinchipe: Abarca un $21 \%$ de su territorio con altitudes entre 2000 a 3800 msnm. Es la parte más despoblada y con relieve muy accidentado, y con elevaciones montañosas y cordilleras, la mayor parte es en territorio ecuatoriano. La temperatura va entre los 15-19 ${ }^{\circ} \mathrm{C}$ y la precipitación entre 1200-3500 mm/año.

b) Cuenca Media del Chinchipe: Es la parte más extensa de la cuenca, abarca un 45\% de su territorio con altitudes entre 1200 a 2000 msnm. Esta zona tiene condiciones climáticas favorables para las actividades agropecuarias, la mayor parte está en territorio peruano, principalmente en la provincia de San Ignacio (Cajamarca). La temperatura entre $20-22{ }^{\circ} \mathrm{C}$ y una precipitación

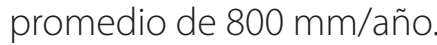

c) Cuenca Baja del Chinchipe: Abarca un 34\% de su territorio con altitudes entre 500 a 1200 msnm. Se ubica netamente en territorio peruano, alberga importante proporción de la población, y corresponde a zonas calurosas con temperaturas entre $25-32{ }^{\circ} \mathrm{C}$. En esta zona de la cuenca se ubican franjas de tierras (valles) con suelos profundos y muy fértiles y con infraestructura de riego mayor.

\section{Flora y fauna representativa}

El territorio de la cuenca del Chinchipe se ubica en parte del área del Parque Nacional de Podocarpus, creado en 1982 por el gobierno ecuatoriano, y en el lado peruano parcialmente en el área del Santuario Nacional Tabaconas Namballe (SNTN). El SNTN creado el 20 de mayo de 1988, es uno de los nueve santuarios del Sistema Nacional de Areas Naturales Protegidas por el Estado (SINANPE), con un área de 32124 has (SERNANP, 2020a) cuyo objetivo es conservar una muestra representativa del páramo y proteger los bosques de neblina y las especies en vías de extinción como el oso de anteojos, tapir y los bosques de Podocarpus (SERNANP, 2020b).

En cuanto a la fauna se destaca la presencia del oso de anteojos (Tremarctus ornatos) y el tapir de altura (Tapirus pinchaque) reconocidos como especies amenazadas, los mismos que son motivo de la creación del SNTN. Así, se ha podido citar también alrededor de unas 60 especies de mamíferos como el tigrillo, mono blanco, zorrillo, guatusa, chonto, entre muchos otros. En aves, se ha listado alrededor de 217 especies, de las cuales 186 dentro del SNTN y fuera del santuario 31 especies, entre éstas el gavilán campestre, el gallinazo negro, el gorrión, la lechuza, la pava de monte, y muchas otras especies de aves. En reptiles, se han determinado al menos unas seis especies entre ellos el equis, el coral, el macanche, lagartija, salamandreja (Elliot, 2009).

\section{Geología y suelos}

La cuenca del Chinchipe en la parte del territorio 
peruano, según los estudios del Gobierno Regional de Cajamarca, para la cuenca mediabaja, mayormente se identifica suelos tipos Vertisol, Phaeozem-Vertisol; para la cuenca mediaalta suelos tipo Leptosol, Andosol-Leptosol, Andosol-Regosol, Andosol, Regosol; estos suelos en la cuenca media, mayormente son dedicados al cultivo de café (Germán Alcántara, 2012).

\section{Hidrología y recursos hídricos}

Desde los años 70s al 2000 se instituyó en esta zona el Proyecto Especial Jaén-San IgnacioBagua (PEJSIB), a través del cual para el año
1978 planteó un proyecto de irrigación en esta cuenca llamado proyecto Shumba que abarcaba los valles de los ríos Cochalán y Shumba que son afluentes del río Chinchipe en altitudes entre los 400-800 m.s.n.m. para poner en riego a unas 20000 hectáreas en los distritos de Santa Shumba, Santa Cruz y Bellavista (MINAG, 1978).

Para el año 1982 a través del PEJSIB se identificaron 10 proyecto de irrigación en esta cuenca, estos bordeaban as 14500 hectáreas nuevas de irrigación (MINAG-INAF-PCM, 1982).

\section{Tabla 1}

Proyectos de irrigación en la cuenca Chinchipe identificados por el PEJSIB

\begin{tabular}{llll}
\hline Proyecto & Distrito & Provincia & $\begin{array}{l}\text { Hectáreas } \\
\text { irrigables }\end{array}$ \\
\hline Pampas de Rumiyacu & Jaén & 2500 \\
Guayape & Santa Rosa & Jaén & 1397 \\
Montegrande & Huarango & Jaén & 1278 \\
Perico & Shirinos & San Ignacio & 4170 \\
El Horcón & La Coipa & San Ignacio & 1100 \\
San Francisco & La Coipa & San Ignacio & 1400 \\
La Balsa & Bellavista & Jaén & 1550 \\
La Capilla & Jaén & Jaén & 900 \\
Timaruca & San Ignacio & San Ignacio & 200 \\
\hline
\end{tabular}

Fuente: PEJSIB (MINAG-INAF-PCM, 1982)

Según la Autoridad Nacional del Agua (ANA), a la actualidad, esta cuenca tiene identificados 2 sectores hidráulicos superficiales en clase de sector "B" y tipo de sector "menor" con unos
490 km de canales de derivación, en unos 18 canales de riego; estos sectores hidráulicos son administrados por la Autoridad Local de Agua (ALA) Chinchipe-Chamaya (ANA, 2020).

Tabla 2

Sectores hidrográficos y sus canales de riego identificados en la cuenca Chinchipe

\begin{tabular}{|c|c|c|c|}
\hline Sector Hidráulico & Subsector Hidráulico & Operador & $\begin{array}{c}\text { Longitud de } \\
\text { canal de } \\
\text { derivación }(\mathrm{Km})\end{array}$ \\
\hline Sector Hidráulico & Jaén-Chililique Montegrande- & Junta de Usuarios & 117.64 \\
\hline Menor Chamaya- & Bellavista-Santa Cruz & Sector Hidráulico & \\
\hline $\begin{array}{c}\text { Huancabamba } \\
\text { Clase B }\end{array}$ & Sambimera & Menor San Ignacio & 15.58 \\
\hline Sector Hidráulico & Chinchipe San Ignacio & & 42.10 \\
\hline Menor Chinchipe & Huarango-Zapotal & & 124.43 \\
\hline \multirow[t]{3}{*}{ Clase B } & $\begin{array}{c}\text { Tabaconas-Perico-Cunía } \\
\text { Hawai Cerezal }\end{array}$ & $\begin{array}{l}\text { Junta de Usuarios } \\
\text { Sector Hidráulico }\end{array}$ & 94.60 \\
\hline & $\begin{array}{c}\text { El Triunfo-Shuba alto- } \\
\text { Shumba Bajo-Canana Inguro }\end{array}$ & Menor San Ignacio & 74.74 \\
\hline & $\begin{array}{c}\text { Perlamayo-San Lorenzo- } \\
\text { Chanayacu }\end{array}$ & & 20.10 \\
\hline
\end{tabular}


Caracterización socioeconómica de la cuenca del Chinchipe Aspectos sociales

La población asentada en el territorio de la cuenca del Chinchipe bordea los 158000 pobladores, de los cuales cerca del $90 \%$ corresponde a los poblados de la provincia de San Ignacio. A la actualidad, según el Censo Nacional del INEI 2017 la provincia de San Ignacio tiene 130.620 habitantes, tiene una densidad poblacional de 27,93 habitantes/km2 y una tasa de crecimiento anual del 2,64 \% (INEI, 2018; Llerena et al., 2010). Dentro del territorio de la provincia de San Ignacio se asientan dos comunidades nativas reconocidas oficialmente, de la tribu Aguaruna, las cuales a su vez tienen comunidades nativas anexas (Elliot, 2009).

En cuanto a la educación, a partir del censo nacional del 2017 con los datos de la región Cajamarca, se puede proyectar que un $75 \%$ de la población tendría un nivel primario o menor y un $13 \%$ no tiene ningún nivel de educación (INEI, 2018). En cuanto a la salud, la población en la cuenca tiene un promedio de $47 \%$ de tasa de desnutrición infantil y de $61 \%$ de tasas de mortalidad infantil, siendo muy similar tanto en los poblados de Perú y los poblados de Ecuador. Aún más, en la provincia de San Ignacio un 13\% de la población no tienen ningún tipo de seguro de salud, es decir población muy vulnerable a las enfermedades (Elliot, 2009; INEI, 2018). Respecto del saneamiento en los poblados de la cuenca, el $73 \%$ de la población tiene agua entubada y sólo un $22 \%$ registra alcantarillado. Puntualmente, la provincia de San Ignacio tiene un $71 \%$ de población con agua entubada y un $21 \%$ con alcantarillado (Elliot, 2009).

\section{Aspectos económicos}

El $40 \%$ de la superficie de la cuenca es dedicada a actividades agropecuarias. La actividad agrícola principal es el cultivo de café, principalmente en el territorio de la cuenca media, el $80 \%$ de la población está dedicada al café, adicionalmente se encuentran otros cultivos como plátano, cacao y productos de panllevar; el café, al ser un producto de agroexportación ha tenido un gran avance, tanto a nivel de rendimiento, estando alrededor de 19 quintales/ha (Dilas J., 2013) y algunas zonas en alrededor de 30 quintales/ha; si bien esta producción está por encima del promedio nacional de 14 quintales/ ha (Diaz \& Carmen, 2017) aún estaría por debajo del promedio de producción de países vecinos como Colombia y Brasil. En cuanto a la actividad pecuaria, principalmente es la crianza de ganado vacuno, y en menor cantidad la crianza de cuyes, piscigranjas, entre otras (Llerena et al., 2010).

La provincia de San Ignacio representa el 12.6 $\%$ de la producción cafetalera nacional y junto con la provincia de Jaén conforman el eje cafetalero Cajamarca en el norte del Perú con un 20.9 \% de la producción nacional (Diaz \& Carmen, 2017). Los avances que ha tenido este eje cafetalero puede estar ligado a la creciente ola de organizaciones de productores que se ha venido incrementando en los últimos años. Así, en la provincia de San Ignacio se registran 15 organizaciones cafetaleras que exportan café directamente al mercado internacional, la Cooperativa CENFROCAFE y la Cooperativa Sol \& Café se ubican en la ciudad de Jaén, las cuales se ubican entre los 15 primeros exportadores de café en el Perú (JNC, 2020), además, se pueden encontrar decenas de organizaciones jóvenes, varias de estas que han recibido financiamiento y apoyo estatal a través del programa AGROIDEAS durante los últimos cinco años (AGROIDEAS, 2020). En un análisis para los años 2001-2014 muestra que las organizaciones cafetaleras de este eje cafetalero han logrado captar el $25 \%$ de los fondos de diversos programas como FINCYT, FONDECYT, AGROIDEAS, PNIA (Dilas Jiménez \& Cernaqué Miranda, 2017).

Así también, se ha podido notar que en los últimos años, con el desarrollo de los mercados de cafés especiales, este eje cafetalero ha mejorado mucho su calidad, teniendo productores que ocupan los primeros lugares en las competencias (ACE, 2021). Asimismo, en estas organizaciones cafetaleras se ha promovido el incremento de las áreas de producción con certificación orgánica. Sin embargo, es importante no perder de vista los cuidados y restricciones que este tipo de agricultura demanda (Dilas-Jiménez \& Ascurra- 
Toro, 2020, Dilas-Jiménez et al., 2020).

\section{Propuesta de gestión integrada de la cuenca del Chinchipe en función al tablero de gestión integrada de cuencas Legislación, políticas públicas y otros documentos aplicables en el Perú}

Se realizó una búsqueda minuciosa de las normativas e instrumentos de gestión públicos que regulan y promueven la gestión integrada de las cuencas en el Perú en cuanto a uso y gestión del agua, gestión integrada y organización de cuencas, distintos usos del agua, zona de conservación, administración y gestión del agua, retribución por servicios ecosistémicos, aprovechamiento sostenible del agua, así como políticas y estrategias. Entre las normativas aplicables se puede indicar:

Ley $N^{\circ}$ 30588, Ley de Reforma Constitucional que Reconoce el derecho de acceso al Agua como Derecho Constitucional: Art. Único, que incorpora el artículo $7^{\circ}$-A de la Constitución Política del Perú. "El Estado reconoce el derecho de toda persona a acceder de forma progresiva y universal al agua potable".

Ley N²29338, Ley de Recursos Hídricos: Título Preliminar Art. III. principio 10; Título I Art. 3; Título II Art. 9; Título IV Consejo de cuenca, Art. 24; Capítulo V Organizaciones de Usuarios Art. 26, Art. 28 Junta de usuarios, Art. 29 Comisiones de usuarios, Art. 30 Comités de usuarios y Art. 99 precisa que los instrumentos de planificación del Sistema Nacional de Gestión de Recursos Hídricos.

Ley $N^{\circ}$ 30157, Ley de las Organizaciones de Usuarios de Agua: Art. 3 de las organizaciones de usuarios del agua. Art. 4 sobre la Personería Jurídica precisa que las Juntas de Usuarios. Art. 10 precisa las atribuciones y obligaciones del Consejo Directivo de la Junta de Usuarios. Art. 11 precisa las facultades de supervisión, fiscalización y sanción de las Juntas de Usuarios.

Ley $N^{\circ}$ 30327, Ley de Promoción de las Inversiones para el Crecimiento Económico y el Desarrollo Sostenible: Art. 10 sobre los Títulos habilitantes que se integran a la Certificación Ambiental Global. Numeral 10.2.1 Recursos hídricos a cargo de la Autoridad Nacional de Agua.

Ley $N^{\circ}$ 30640, Ley que modifica la ley 29338, Ley de Recursos Hídricos, mediante el establecimiento de los Criterios Técnicos para la Identificación y Delimitación de las Cabeceras de Cuenca: Art. 2 que modifica el Art. 75 de la Ley 30640, (...) El Estado reconoce como zonas ambientalmente vulnerables las cabeceras de cuenca donde se originan los cursos de agua en una en hidrográfica.

Ley №30215, Ley de mecanismos de retribución por servicios ecosistémicos, y su reglamento aprobado con D.S. N 009-2016-MINAM: en su Art. 6, establece la "regulación hídrica" como uno de los 13 servicios ecosistémicos factibles para establecer Mecanismos de Retribución por Servicios Ecosistémicos (MERESE).

D.S. N ${ }^{\circ}$ 001-2010-AG, Reglamento de la Ley $N^{\circ}$ 29338: Art. 4 sobre la administración del agua y de sus bienes asociados; Art. 6 hace hincapié en la gestión integrada de los recursos hídricos; Art. 11 La ANA es el ente rector del Sistema Nacional de Gestión de los Recursos Hídricos; Art. 18 sobre participación de las comunidades campesinas y comunidades nativas; Art. 43 Sobre las cuencas transfronterizas precisa que el aprovechamiento de aguas en la cuenca transfronteriza.

D.S. $\quad N^{\circ}$ 012-2009-MINAM. Política Nacional del Ambiente: En su Eje de Política 1 sobre Conservación y aprovechamiento sostenible de los recursos naturales y de la diversidad biológica. Así también en su Eje de Política 2 Gestión integral de la calidad ambiental.

Política y Estrategia Nacional de Recursos Hídricos: Dentro del Eje de Política 1 Gestión de la cantidad, en la estrategia de intervención 1.1 entre otros, se tiene el lineamiento de normar, regular la protección y planificación de los recursos hídricos en las cuencas hidrográficas; En el eje de Política 3 estrategia 3.1 se presentan los lineamientos para la implementación de la gestión integrada de los recursos hídricos por 
cuenca.

R.J. 575-2010-ANA, Lineamientos Generales para la Creación de Consejos de Recursos Hídricos de la Cuenca: Precisa que el proceso de conformación y creación de un Consejo de Recursos Hídricos de la Cuenca, es un proceso planificado por lo que debe seguir las siguientes etapas: 1) Preparatoria y de coordinación interinstitucional, 2) Caracterización general de la cuenca e identificación de actores, 3) Conformación y acreditación de representantes, 4) Preparación del expediente de creación, y 5) Creación del Consejo de Recursos Hídricos de Cuenca.

\section{Identificación de actores en la cuenca}

Los actores de una cuenca son personas naturales y/o jurídicas que intervienen en la gestión de los recursos hídricos en el ámbito de influencia de una cuenca hidrográfica (Resolución Jefatural NN 575-2010, Lineamientos Generales Para La Creación de Consejos de Recursos Hídricos de La Cuenca, 2010).

De manera preliminar se hizo una identificación de actores para la cuenca del Chinchipe encontrándose los siguientes (Llerena et al., 2010, (ANA, 2011, ANA, 2020a, ANA, 2020b, EPSMarañón, 2020, JNC, 2020, GORE-Cajamarca, 2020, SERNANP, 2020b): (1) Actores endógenos: ONG Caritas, Vicariato Apostólico Francisco Javier del Marañón y Santuario Nacional Tabaconas Namballe (SNTN). (2) Actores exógenos: Proyecto Bosques del Chinchipe. (3) Actores directos: Juntas de Usuarios, Comités de Usuarios, Consejo de Recursos Hídricos de la Cuenca (no constituido), Comités o Asociaciones de Productores. (4) Actores indirectos: ONGs, Universidad Nacional de Jaén, Universidad Nacional de Cajamarca-Sede Jaén, Institutos Superiores en Jaén y San Ignacio. (5) Actores del sector público: Autoridad Nacional del Agua, Autoridad Local de Agua Chinchipe-Chamaya, Gobierno Regional de Cajamarca-Dirección Regional de Agricultura, Gobiernos Regionales de Amazonas y Piura, Municipalidad Provincial de San Ignacio, Municipalidad Provincial de Jaén, Entidad Prestadora de Servicios "Marañon" S.A. (6) Actores privados: Empresas comercializadoras, entidades financieras, comunidades nativas.

\section{Problemas identificados en la cuenca}

Dada la tipología especial de la cuenca por ser transfronteriza según la información sistematizada, esta cuenca aborda diversos problemas que se pueden resumir en los siguientes (ANA, 2011, 2020a; Dilas J., 2013; Llerena et al., 2010; MINAM, 2020): (1) Legales e institucionales: ligados a que la cuenca no cuenta con un Consejo de Recursos Hídricos, así como las dificultades de la ALA. (2) Administrativos y de gestión: Debidos a la característica transfronteriza y multi-regional de la cuenca. (3) Antrópicos y uso del agua: Vinculados a la deforestación, el deficiente uso del agua, la contaminación de aguas por vertimientos, la erosión y degradación de suelos y la quema de laderas. (4) Ambientales y naturales: Relacionados al fenómeno del niño y a que no se tiene sistema de pagos por servicios ambientales. (5) Infraestructura y vías: Deficiencia en infraestructura para cruzar el río Chinchipe. (6) Económicos y financieros: Vinculados a la baja recaudación de las organizaciones de usuarios del agua de riego, los bajos ingresos de las familias y problemas financieros.

\section{Escenario deseado propuesto}

Entre las perspectivas de un escenario deseado para la cuenca del rio Chinchipe, puntualmente en el territorio peruano, se podría indicar lo siguiente:

a) En organización y gestión: Que se conforme y funcione el Consejo de Recursos Hídricos de la cuenca del Chinchipe, y a través del cual se implemente un sistema de gestión integral de la cuenca con al apoyo de los actores que correspondan. Asimismo, que exista un liderazgo del GORE Cajamarca y de los gobiernos locales en cada sector, para el desarrollo de proyectos sociales y productivos con enfoque de cuenca.

b) En lo económico y social: Que la población de la cuenca esté en una situación de mejor nivel económico con una economía sostenible, un sector agropecuario y empresarial sobresaliente con actores que colaboran para contribuir a ello. También, que usuarios del agua desde el uso doméstico, el uso industrial y principalmente el 
uso agropecuario, cumplan con sus pagos que permitan hacer inversiones de mejora en las infraestructuras de riego.

c) En lo ambiental: Velar por un uso responsable y sostenible del agua, que permita asegurar aprovisionamiento y calidad del recurso. Así también, que los pobladores, las municipalidades provinciales y distritales, así como otros actores como el SNTN y las universidades contribuyan en la recuperación de sistemas boscosos y la conservación de los bosques existentes. Asimismo, que la agricultura se desarrolle bajo sistemas sostenibles, con tecnologías de bajo impacto en el ambiente y principalmente en la conservación del suelo, como en el adecuado y eficiente uso del agua.

\section{Instrumentos de gestión y gobernanza de la cuenca}

Entre los instrumentos de gestión que debería promoverse son: 1) La elaboración de presupuestos de desarrollo concertado de los Gobiernos Locales con enfoque de cuenca; 2) Que los Planes Estratégicos Multianuales, tanto de los Gobiernos Regionales como de los Gobiernos Locales, propongan proyectos integrales con enfoque de cuenca que aseguren un desarrollo sostenible del territorio; 3) Que se cuente con un Plan Estratégico para la gestión adecuada y eficiente de los recursos hídricos de la cuenca Chinchipe.

Para la adecuada gobernanza de la cuenca y en atención a la legislación nacional, se debe: 1) Tener conformado y en funcionamiento el Consejo de Recursos Hídricos de la cuenca del Chinchipe; 2) Tener una ALA empoderada y con un sistema bien organizado de las organizaciones de usuarios (comités, comisiones, juntas); 3) Que las Agencias Agrarias y las Oficinas que trabajan por el agro en las municipalidades apoyen a las acciones de la ALA.

\section{Soluciones identificadas para su análisis y futura implementación}

Dado el análisis de problemas presentado en el cuadro 5, se puede proponer las siguientes alternativas de solución, según agrupamiento del problema, así:

a) Legaleseinstitucionales:Urgente conformación y funcionamiento del Consejo de Recursos Hídricos de la cuenca del Chinchipe. Así también el empoderamiento y mayor financiamiento a la Autoridad Local del Agua en la cuenca.

b) Administrativos y de gestión: Conformación de una mancomunidad regional en torno a la cuenca, con el liderazgo del GORE Cajamarca y el apoyo de los GOREs Piura y Amazonas.

c) Antrópicos y uso del agua: 1) Reforestación y conservación de bosques a través de la elaboración y ejecución de proyectos de reforestación, principalmente en zonas de la cuenca media y cuenca alta. Para la cuenca mediaalta: Recomendable la siembra y conservación de los Romerillos (Prumnophytis sp., Nageia sp.), Cascarilla (Cinchona sp.), Laurel (Cordia alliodora), además de otras especies propias de la zona como especies de lauráceas (robles), myrtaceas, melastomatáceas y moráceas, que son las familias más abundantes en estas zonas (Dilas-Jiménez, 2009). 2) Provisión de mayor presupuesto o apoyo a las labores de cuidado de bosques en el área protegida del SNTN, clave para proteger las zonas de cabeza de cuenca. 3) Promoción y apoyo al cultivo de café, cacao y otros cultivos bajo sistemas agroforestales que aseguren una mejor sostenibilidad de uso del suelo y del agua. 4) Actividades para el eficiente uso del agua. 5) Actividades para evitar la contaminación de aguas. 6) Conservación de suelos y fertilidad: Implementar técnicas para la adecuada conservación y fertilidad de suelos, principalmente en el cultivo de café en la cuenca media.

d) Ambientales y naturales: Implementación de mecanismos de retribución MERESE por la EPS Marañón en las provincias de Jaén y San Ignacio bajo las normativas establecidas por el MINAM y la SUNASS. Con ello, la ejecución de proyectos productivos y de conservación en la cuenca media y alta, más aún, cuando estos bosques montanos de neblina son ecosistemas boscosos biodiversos y ecoeficientes (Dilas-Jiménez \& Huamán Jiménez, 2020). 
e) Infraestructura y vías: Implementación de proyectos de infraestructura vial en el rio Chinchipe que facilite el traslado para ambas márgenes del rio sin ocasionar peligros para los transeúntes.

f) Económicos y financieros: 1) Empoderar a las organizaciones de usuarios del agua. 2) Implementar programas para la mejora de los ingresos de las familias dedicadas a la agricultura: Tecnologías de producción agrícola, generar mecanismos de garantías que facilite el acceso a financiamiento para pequeños agricultores en la cuenca.

\section{Apreciaciones críticas sobre la propuesta}

En cuanto a la aplicación del tablero de gestión integrada de cuencas propuesto por Dourojeanni $(2000,2020)$ y que se ha desarrollado como parte de este documento, éste es factible de aplicar como herramienta para la gestión integrada de una cuenca. Asimismo, esta metodología es acorde a otras más recientes como el enfoque sistémico de gestión integrada de cuencas propuesto por Lee et al. (2008), quien considera seis puntos básicos para la gestión: 1) Comprender los componentes así como los procesos que ocurren en una cuenca; 2) Identificar y hacer una clasificación de los problemas a resolver, 3 ) Establecer metas que sean claras y específicas; 4) Proponer una lista de opciones para el manejo de la cuenca; 5) Analizar y eliminar las opciones que sean inviables de implementar; y 6) Hacer pruebas de efectividad de las opciones que sean factibles de implementar.

En el desarrollo del tablero de gestión, se ha determinado que una debilidad clave a resolver es el fortalecimiento de la gobernabilidad a través de la conformación de Consejo de Recursos Hídricos de la Cuenca como un órgano intermediario de escala regional, que permita abordar las necesidades de la cueca y su adecuada gestión, este modelo además es respaldado por estudios realizados en el sur peruano (Mancilla García \& Bodin, 2019; Popovici et al., 2021). Sin embargo, la participación activa de los usuarios y actores en la cuenca permitirá implementar una gestión eficaz de la cuenca (Speer, 2012).
En este sentido, se ha hecho énfasis en el fortalecimiento de la asociatividad; sin embargo, esto no ha sido muy desarrollado en la cuenca baja, como si lo ha sido en las zonas cafetaleras ubicadas en la cuenca media-alta, donde se han formado muchas asociaciones y cooperativas de cafetaleros (Dilas J., 2013), encontrándose varias de estas con buen nivel de desarrollo como organizaciones cafetaleras exportadoras de café (JNC, 2020).

Asimismo, es importante la acción que deben tomas las universidades en la zona para cubrir vacíos de conocimiento, a la actualidad por ejemplo se están haciendo investigaciones en la cuenca cuanto a los bosques estacionalmente secos (Marcelo Peña, 2013). Finalmente, entre las soluciones para problemas de uso antrópico se ha propuesto la estrategia de hacer agricultura a través de Sistemas Agroforestales buscando el beneficio del suelo (Dilas-Jiménez \& MugruzaVassallo, 2020).

\section{CONCLUSIONES}

Para la implementación de un enfoque integrado de gestión de cuencas es necesario la interacción y participación activa de los actores involucrados, haciendo uso de instrumentos de gestión un sistema de gobernanza robusto.

La puesta en práctica del tablero de gestión integrada de cuencas es adecuado y adaptable para la gestión de la cuenca transfronteriza del Chinchipe ubicada en el norte del Perú, puesto que ha permitido llegar a visualizar escenarios deseados, proponer instrumentos de gestión aplicables e identificar propuestas de solución.

Entre las propuestas de solución identificadas para la adecuada gestión integrada de la cuenca del Chinchipe se destaca como clave la conformación y funcionamiento del Consejo de Recursos Hídricos de la cuenca; así como, el empoderamiento de la Autoridad Local del Agua y de las organizaciones de usuarios del agua, la activa integración de los actores, el fortalecimiento de la asociatividad en los productores, la reforestación e implementación de Sistemas Agroforestales, entre otras. 


\section{REFERENCIAS BIBLIOGRAFICAS}

ACE. (2021). COE Auction Results. Disponible en: https:// allianceforcoffeeexcellence.org/ competition-auction-results/

AGROIDEAS. (2020). Lista de planes de negocio aprobados 2010-2020. Disponible en: https://www.agroideas.gob.pe/wpcontent/uploads/2020/05/RELACIONDE-ORGANIZACIONES-INCENTIVOASOCIATIVIDAD.pdf

Aguirre, M. (2011). La cuenca hidrográfica en la gestión integrada de los recursos hídricos. Revista Virtual REDESMA, 5(1). Disponible en: http://www.siagua.org/sites/default/files/ documentos/documentos/cuencas_m_ aguirre.pdf

ANA. (2009). Mapa hidrográfico del Perú. Disponible en: https://www.ana.gob.pe/ sites/default/files/normatividad/files/ mapa_hidrografico_0_0_2.pdf

Resolución Jefatural № 575-2010, Lineamientos Generales para la Creación de Consejos de Recursos Hídricos de la Cuenca, (2010). Disponible en: https://www.ana.gob.pe/ normatividad/lineamientos-generalespara-la-creacion-de-consejos-de-recursoshidricos-de-cuenca-0

ANA. (2011). Diagnóstico de problemas y conflictos en la gestión de los recursos hídricos en la cuenca Chinchipe-Chamaya. Disponible en: http://repositorio.ana.gob. pe/handle/20.500.12543/44

ANA. (2015). Política y Estrategia Nacional de Recursos Hídricos. Disponible en: https:// www.ana.gob.pe/sites/default/files/ default_images/politica_y_estrategia_ nacional_de_recursos_hidricos_ana.pdf

ANA. (2020a). Compendio delimitación de los sectores hidráulicos a nivel nacional: VI Autoridad Administrativa del Agua Marañón (Sectores y subsectores hidráulicos).
Disponible en: http://repositorio.ana.gob. pe/handle/20.500.12543/4686

ANA. (2020b). Consejos de Recursos Hídricos de Cuenca. Disponible en: https://www. ana.gob.pe/nosotros/planificacion-hidrica/ plan-gestion-cuencas.

Carranza, J. (2011). Evaluación hidrológica de las cuencas amazónicas peruanas.

CEPAL. (1982). Manejo de cuencas y desarrollo de zonas altas en América Latina. Disponible en: https://centroderecursos.cultura.pe/ sites/default/files/rb/pdf/Evaluacion\%20 hidrologica\%20de\%20las\%20cuencas\%20 amazonicas\%20peruanas.pdf

Diaz, C., \& Carmen, M. (2017). Linea de base del café en el Perú. Disponible en: file:///C:/ Users/Usuario/ Downloads/Libro cafe_ PNUD_PE.pdf

Dilas-Jiménez, J. O. (2009). Diversidad, composición, estructura y distribución espacial arbórea de un área de bosques de neblina, Jaén, Perú, 2008. Universidad Nacional de Cajamarca.

Dilas-Jiménez, J. O., \& Ascurra-Toro, D. (2020). Agroecología: Una alternativa sostenible para la pequeña agricultura en un escenario post COVID19. Revista de Investigación Científica y Tecnológica Llamkasun, 1(2). doi: https://doi.org/https//doi.org/10.47797/ llamkasun.v1i2.9

Dilas-Jiménez, J. O., \& Huamán Jiménez, A. O. (2020). Captura de carbono por un bosque montano de neblina del Perú. Revista de Investigación Científica y Tecnológica Alpha Centauri, 1(3), 13-25. doi: https://doi.org/ https://doi.org/10.47422/ac.v1i3.16

Dilas-Jiménez, J. O., \& Mugruza-Vassallo, C. (2020). Instalación De Fincas Cafetaleras En Sistema Agroforestal Para Recuperación 
Y Sostenibilidad De Suelos Degradados De Selva Alta. Revista de Investigación En Agroproducción Sustentable, 4(1). Disponible en: http://revistas.untrm.edu.pe/ index.php/INDESDOS/article/view/534

Dilas-Jiménez, J., Zapata-Ruiz, D., Arce-Almenara, M., Ascurra-Toro, D., \& Mugruza-Vassallo, C. (2020). Análisis comparativo de los costos de producción y rentabilidad de los cafés especiales con certificación orgánica y sin certificación. South Sustainability, 1(2), 1-10. doi: https://doi.org/10.21142/SS-01022020-017

Dilas J.,J.O.(2013). Identificación deoportunidades de innovación tecnológicas en la producción del café en la región Cajamarca [Universidad Peruana Cayetano Heredia]. Disponible en: http://repositorio.upch.edu.pe/handle/ upch/1315

Dilas Jiménez, J. O., \& Cernaqué Miranda, O. C. (2017). El sector cafetalero peruano: Un enfoque a la CTI para su competitividad (F. Editorial (ed.); Primera Ed). Universidad Continental.

Dourojeanni, A. (1994). Políticas públicas para el desarrollo sustentable: La gestión integrada de cuencas. Disponibleen: https:// repositorio.cepal.org/handle/11362/19210

Dourojeanni, A. (2000). Procedimientos de gestión para el desarrollo sustentable (CEPAL (ed.); Primera Ed). Naciones Unidas. https:// repositorio.cepal.org/handle/11362/5564

Dourojeanni, A. (2020). Gestión de intervenciones sobre los recursos hídricos: Elementos de gestión y terminologías.

Dourojeanni, A., Jouravlev, A., \& Chávez, G. (2002). Gestión del agua a nivel de cuenca: teoría y práctica (CEPAL (ed.); Primera Ed). Naciones Unidas. Disponible en: https:// repositorio.cepal.org/handle/11362/6407

Elliot, J. (2009). Los bosques de la cuenca transfronteriza del rio Mayo-Chinchipe Perú-Ecuador (ITDG (ed.); Primera Ed). Soluciones Prácticas ITDG. Disponible en: $\quad$ http://infobosques.com/portal/wpcontent/uploads/2017/09/Los-bosquesde-la-cuenca-transfronteriza-del-río-MayoChinchipe.pdf

EPS-Marañón. (2020). EPS Marañón localidades de influencia. Disponible en: http:// epsmaranon.com.pe/nosotros

Faustino, J., \& Jiménez, F. (2000). Manejo de cuencas hidrográficas. Disponible en: $\quad$ http://201.207.189.89/bitstream/ handle/11554/2946/Manejo_de_cuencas_ hidrograficas.pdf? sequence=1\&isAllowed=y

Felipe-Morales, C. (2007). Gestión integrada de cuencas hidrográficas. Germán Alcántara. (2012) Mapa de suelos. Disponible en: https://zeeot.regioncajamarca.gob.pe/ sites/default/files/M12 Suelos.pdf

GORE-Cajamarca. (2020). Agencias Agrarias Cajamarca. Disponible en: http://www. agriculturacajamarca.gob.pe/portal/ $\mathrm{mn} / 212$

INEI. (2018). Perú resultados definitivos de los censos nacionales 2017. Tomo I, Tomo II. Disponible en: https://www.inei.gob.pe/ media/MenuRecursivo/publicaciones_ digitales/Est/Lib1544/

JNC. (2020). Perú: Exportación de café enerodiciembre 2019 por empresa. Estadísticas - Junta Nacional del Café. Disponible en: https://juntadelcafe.org.pe/estadisticas/

Lee, K. S., Chung, E. S., \& Kim, Y. O. (2008). Integrated watershed management for mitigating streamflow depletion in an urbanized watershed in Korea. Physics and Chemistry of the Earth, 33(5), 382-394. doi: https://doi.org/10.1016/j.pce.2008.02.033

Llerena, C., Cruz-Burga, Z., Durt, É., MarceloPeña, J., Martinez, K., \& Ocaña, J. (2010). Gestión ambiental de un ecosistema frágil. 
Los bosques nublados de San Ignacio, Cajamarca, cuenca del rio Chinchipe (ITDG (ed.); Primera Ed). Soluciones Prácticas ITDG. Disponible en: http://infobosques. com/portal/wp-content/uploads/2017/09/ Gestión-ambiental-de-un-ecosistema-frágil. pdf

Mancilla García, M., \& Bodin, Ö. (2019). Participatory Water Basin Councils in Peru and Brazil: Expert discourses as means and barriers to inclusion. Global Environmental Change, 55(January), 139-148. doi: https:// doi.org/10.1016/j.gloenvcha.2019.02.005

Marcelo Peña, J. L. (2013). Vegetación leñosa, endemismos y estado de conservación en los bosques estacionalmente secos de Jaén, Perú. Revista Peruana de Biología, 15(1), 43-52. doi: https://doi.org/10.15381/rpb. v15i1.1669

MINAG-INAF-PCM.(1982).Inventario de proyectos de riego en el ámbito del proyectos especial Jaén-San Ignacio-Bagua (PEJSIB). Disponible en: http://repositorio.ana.gob. pe/handle/20.500.12543/1671

MINAG. (1978). Irrigación de los valles de Chinchipe, Utcubamba y Marañon (Zona de Jaén): Proyecto Magunchal - Proyecto Shumba. Disponible en: http://repositorio. ana.gob.pe/handle/20.500.12543/3937

MINAM. (2020). Avances de MERESE con Empresas Prestadoras. Disponible en: https://www.minam.gob.pe/economia-yfinanciamiento-ambiental/mecanismosde-retribucion-por-servicios-ecosistemicosmrse/

Popovici, R., Erwin, A., Ma, Z., Prokopy, L. S., Zanotti, L., Bocardo Delgado, E. F., Pinto Cáceres, J. P., Zeballos Zeballos, E., Salas O'Brien, E. P., Bowling, L. C., \& Arce Larrea, G. R. (2021). Outsourcing governance in Peru's integrated water resources management. Land Use Policy, 101. doi: https://doi.org/10.1016/j. landusepol.2020.105105
SERNANP. (2020a). Areas Naturales Protegidas de Administración Nacional con Categoría Definitiva. Disponible en: $\quad$ https://www.sernanp.gob.pe/ documents/10181/2872611/Listado_ ANP_28-08-2020/ddc00c79-df75-4d22bada-5cda642a5b3f

SERNANP. (2020b). Santurario Nacional Tabaconas Namballe. Disponible en: https://www. sernanp.gob.pe/tabaconas-namballe

Speer, J. (2012). Participatory Governance Reform: A Good Strategy for Increasing Government Responsiveness and Improving Public Services? World Development, 40(12), 2379-2398. doi: https://doi.org/10.1016/j. worlddev.2012.05.034

Vasquez, C. (2011). Estudio hidrológico de la región Cajamarca. Disponible en: https:// zeeot.regioncajamarca.gob.pe/sites/ default/files/HIDROLOGIA.pdf 